\title{
Divergierende Lohn- und Inflationsentwicklungen im Euroraum: Ursachen und Folgen
}

Der Blick zurück auf zehn Jahre mit einer gemeinsamen Währung in weiten Teilen Europas ergibt ein widersprüchliches Bild. Einerseits füllte der Euro seine Rolle als internationale Anlagewährung zunehmend stärker aus, und das Inflationsziel der Europäischen Zentralbank (EZB) wurde für den Euroraum insgesamt weitgehend erreicht. Andererseits ging dies einher mit einem deutlichen, von vielen Ökonomen in dieser Form nicht erwarteten und über Jahre anhaltenden Auseinanderlaufen der nationalen Inflations- und Lohnstückkostenentwicklungen. Ursachen, Folgen und Schlussfolgerungen dieser Entwicklungen werden im Folgenden diskutiert.

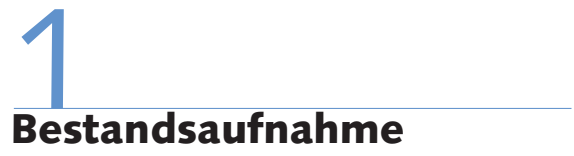

Auffällig im Euroraum ist die hohe Persistenz der Inflationsratenabstände zum Durchschnitt der Europäischen Währungsunion (EWU). Ländern wie Spanien und Griechenland mit dauerhaft überdurchschnittlichen Inflationsraten stehen Deutschland, Frankreich und Österreich gegenüber, deren Raten die meiste Zeit unter dem Durchschnitt lagen. Abbildung 1 zeigt die Entwicklung ausgewählter Inflationsraten im Euroraum.

Insbesondere in der Phase des konjunkturellen Abschwungs, die mit dem Platzen der New-Economy-Spekulationen nach dem Jahre 2001 einsetzte, kam es zu einer lebhaften Diskussion über die Sprengkraft, die die starken Divergenzen für den Zusammenhalt der Währungsunion haben könnten (Gros 2006; Münchau 2006; Prior-Wandesforde/Hacche 2005). Dabei wurde vor allem problematisiert, dass nominale und realwirtschaftliche Entwicklungen miteinander verknüpft sind und es zu einem sich aufschaukelnden und anhaltend divergierenden Prozess kommen könnte (Lane 2006; Belke/Gros 2007; Geiger/Spahn 2007). Die persistenten Unterschiede hinsichtlich der Höhe gesamtwirtschaftlicher Lohnzuwächse und der Produktivitätszunahme - zusammengefasst in der Größe der Lohnstückkosten - waren dabei als eine wichtige Ursache dieser Entwicklung benannt worden. Diese Auffassung wurde auch von der Europäischen Zentralbank (EZB) geteilt, wie es der Präsident der EZB im Jahr 2006 formulierte: „In most countries (des Euroraums), domestic factors dominate external factors in generating inflation differentials. In particular, we have witnessed a sustained divergence

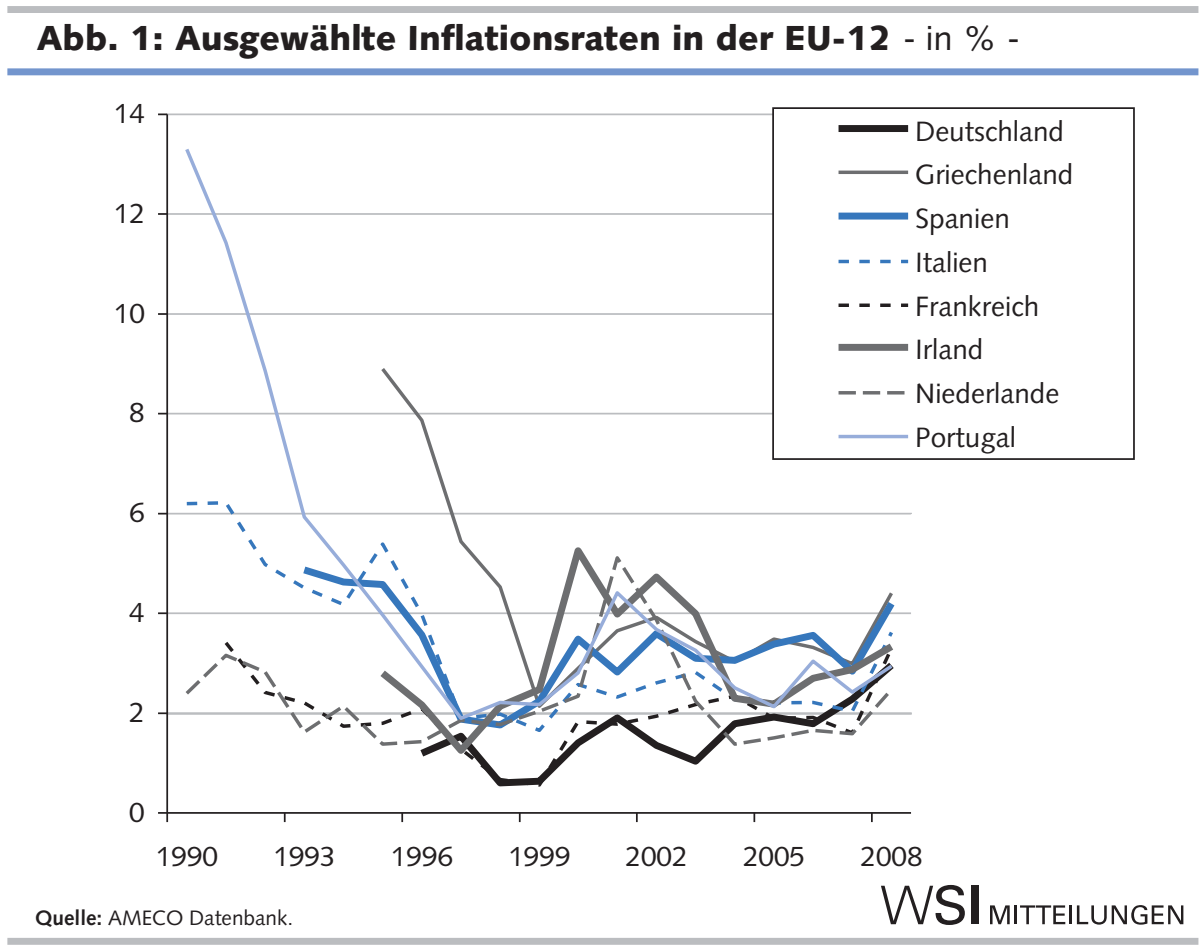

of wage developments across the euro area, and narrower differences in labour productivity growth. As a result, differentials in the growth of unit labour costs have been persistent" (Trichet 2006).

Abbildung 2 zeigt die Entwicklung des relativen nominalen Lohnstückkostenindex (relativ zum Euroraum, $1999=100)$ für ausgewählte Länder des Euroraums. Der Index kann als realer Wechselkurs in Lohnstückkosten, gemessen relativ zum Durchschnitt des Währungsraumes, gelesen werden. Der Wert des hier verwendeten Index für ein spezifisches Jahr kann zudem als Maß für die prozentuale Abweichung des realen Wechselkurses bezogen auf das Basisjahr dienen. Ein Wert von 109,1 für Spanien für das Jahr 2005 würde damit eine reale Aufwertung für das Land um 9,1 \% im Zeitraum 1999 bis 2005 bedeuten. Über den längeren Zeitraum 1999-2008 verläuft dabei - spiegelbildlich zu Abbildung 1 die Entwicklung tendenziell „bipolar“. So weisen viele EU-Länder überdurchschnittlich steigende nominale Lohnstückkosten auf. Das bedeutet eine Verteuerung der heimischen Produktion relativ zum Durchre Länder wie Deutschland und Österreich gegenüber, die relativ zum Durchschnitt des Euroraums real abwerteten.

Ihre Entsprechung findet diese Verschiebung der realen Wechselkurse in einem seit Jahren größer werdenden Auseinanderklaffen der nationalen Leistungsbilanzdefizite (Abbildung 3). Zweistelligen

Ulrich Fritsche, Dr., ist Juniorprofessor an der Universität Hamburg. Arbeitsschwerpunkt: Empirische Wirtschaftsforschung. e-mail: ulrich.fritsche@wiso.uni-hamburg.de. schnitt des Euroraums. Dem stehen ande- 


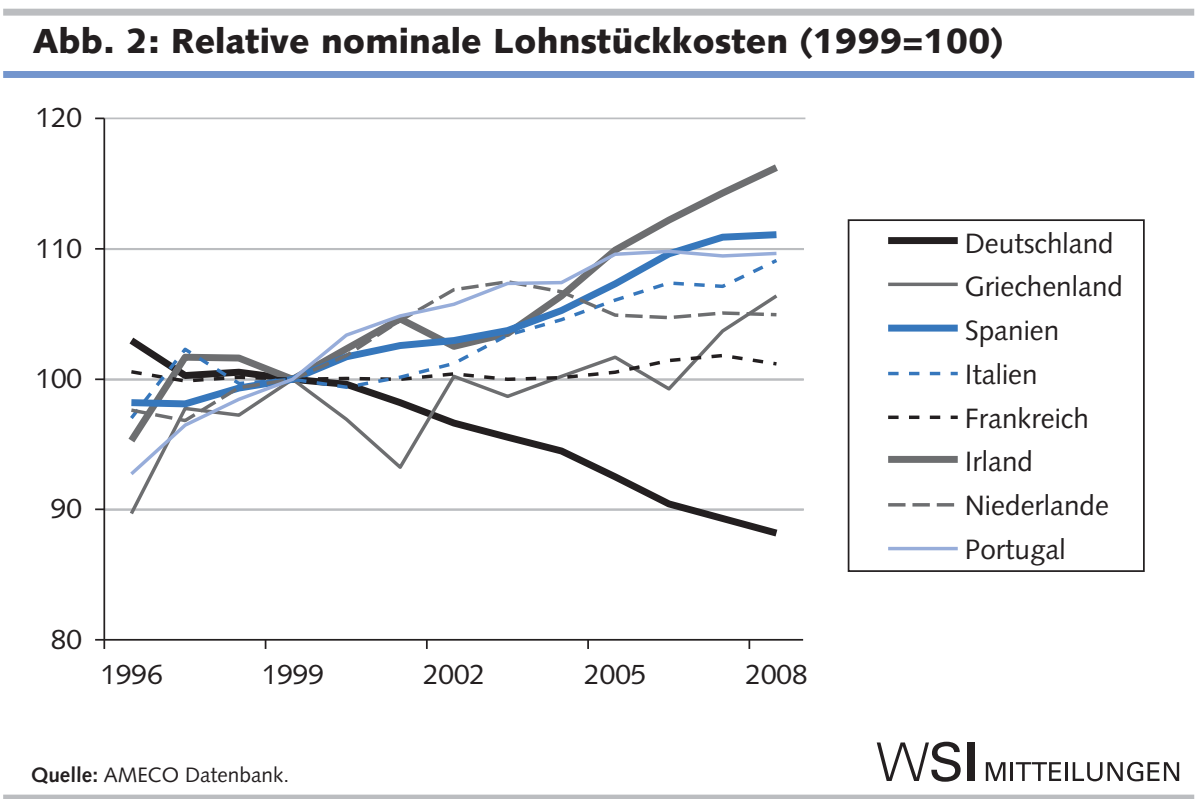

Abb. 3: Leistungsbilanz 2008 - in \% des BIP -

$-12,7$

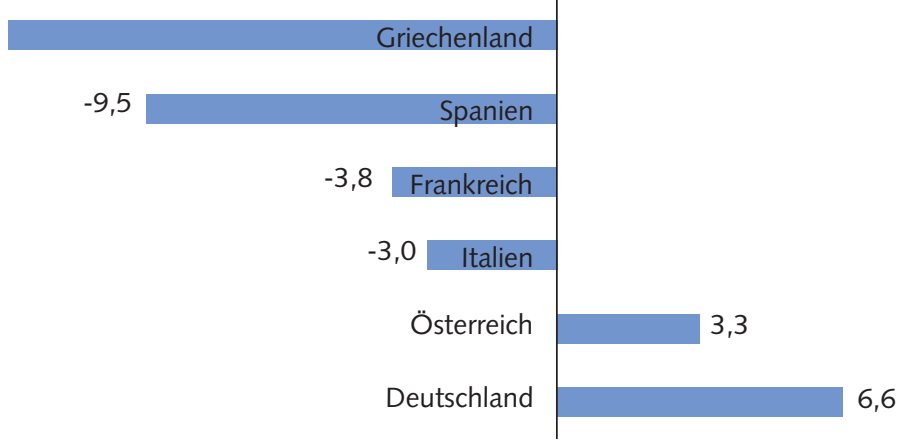

Quelle: Eurostat.

aktuellen Leistungsbilanzdefiziten in Griechenland, Spanien und Portugal stehen deutliche Überschüsse in Deutschland und den Niederlanden gegenüber.

Im Folgenden wird deshalb diskutiert, wie diese Divergenzen zustande kommen und wie sie in ihren Auswirkungen auf die Währungsunion und innerhalb dieses Bereiches auf die betroffenen Länder zu bewerten sind (Abschnitt 2). Anschließend (Abschnitt 3) werden Schlussfolgerungen für den daraus resultierenden wirtschaftspolitischen Handlungsbedarf gezogen.

\section{2 \\ Ursachen und Folgen der Divergenzen}

Innerhalb einer Währungsunion besagt eine phasenweise hohe Inflationsratendi-
WSI MITTEILUNGEN

lang wieder verschwinden. Dafür sorgt, dass es neben dem kurz- und mittelfristig auf eine steigende Divergenz hinwirkenden Realzinskanal auch immer einen Kanal des realen Wechselkurses gibt, der die divergente Entwicklung korrigieren sollte. Allerdings wirken diese Mechanismen, wie im Folgenden erläutert wird, nicht immer reibungslos und vor allem sind die Anpassungszeiten länger, als zu Beginn der Währungsunion von vielen erwartet worden war (DG ECFIN 2008).

Vielfach wird zur Erklärung der geschilderten Divergenzen argumentiert, die Existenz der Europäischen Währungsunion verstärke und verlängere die nationalen Konjunkturzyklen, weil keine nationale Geldpolitik mehr existiert, die auf nationaler Ebene entgegensteuern könnte (Lane 2006; Dullien/Schwarzer 2005). Da der kurzfristige Nominalzins für die Währungsunion insgesamt von der EZB gesetzt wird und sich die Risikoaufschläge auf langfristige Nominalzinsen üblicherweise in der Währungsunion im engen Rahmen halten (zumindest bis vor Kurzem), kann sich ein Boom oder ein Abschwung in einem einzelnen Land selbst verstärken. Da in einem Aufschwung in der Regel die Inflation anzieht, fällt dabei der nationale Realzins. Dieser „Realzinseffekt“ kurbelt die Wirtschaft weiter an, vor allem über ein stärkeres Wachstum der Ausrüstungs- und Wohnungsbauinvestitionen (und des privaten Konsums infolge von Vermögenseffekten). ${ }^{1}$

Dem steht negativ gegenüber, dass das betroffene Land gleichzeitig Wettbewerbsfähigkeit einbüßt (Kanal des „realen Wechselkurses"); da diese Verschlechterung im Außenhandel aber im Allgemeinen deutlich langsamer wirkt als der Effekt des Realzinskanals auf Investitionen und Konsum, kann sich ein nationaler Boom in der Währungsunion gegenüber der Situation mit einer eigenen nationalen Zentralbank deutlich verlängern und zu einem insgesamt wesentlich stärkeren Verlust an Wettbewerbsfähigkeit führen. Der Wettbewerbseffekt sollte langfristig dafür sorgen, dass sich die durch divergierende Realzinsen ausgelösten Abweichungen wieder abbauen, vor allem, da in einer Währungsunion mit unverrückbar festen nominalen stabilitätsorientierter Politik sein.

Klar ist jedoch: Wenn Faktor- und Produktmärkte zum Gleichgewicht tendieren - wie theoretisch postuliert wird - und die Lohnpolitik stabilitätsgerecht agiert, dann sollten solche Differenzen über kurz oder
1 In modernen makroökonomischen Modellen wird die Wirkung des Realzinses direkt auf den Konsum betrachtet. Empirisch ist die Stärke dieses Effektes mindestens umstritten (Campbell/Mankiw 1989). 
Wechselkursen jede kurzfristige Änderung der nationalen Inflationsrate den realen Wechselkurs dauerhaft ändert.

Spiegelbildlich sieht es in einer Phase schwachen Wachstums aus: Da die Inflationsrate hinter jener im Durchschnitt des Euroraums zurückbleibt, steigt jetzt der relevante Realzins und das ohnehin schwache Wachstum wird zusätzlich gedämpft. Zwar verbessert sich gleichzeitig auch die Wettbewerbsfähigkeit; bis daraus allerdings ein ausreichend großer Impuls erwächst, um die Krise zu beenden, vergeht eine deutlich längere Zeit als in einer Situation, in der eine nationale Notenbank schnell und entschieden den Zins senken könnte, um das nationale Wachstum anzukurbeln.

Auch wenn die Preisentwicklung angesichts der geschilderten Prozesse für einige Zeit in verschiedenen Ländern unterschiedlich wäre, so müssen dadurch nicht unbedingt anhaltend negative Folgen für realwirtschaftliche Prozesse erwachsen. Man könnte, wie vor allem in den ersten Jahren nach der Einführung der gemeinsamen Währung vielfach üblich (Alesina et al. 2001; Issing 2002), argumentieren, dass im Durchschnitt Arbeitslosigkeit und Wirtschaftsleistung nicht beeinflusst werden - sofern es keine Störung in der wirtschaftspolitischen Aufgabenteilung zwischen Geld-, Fiskal- und Lohnpolitik gibt. Unter der Annahme, dass letztlich die Anpassung des realen Wechselkurses zu einer Korrektur von Divergenzen führt, ergibt sich dann eine konjunkturpolitische Aufgabenteilung, die von Allsopp/Artis in einer Rückschau auf die ersten Jahre der Währungsunion als ",consensus or official view“ charakterisiert wurde: „First, price stability is the responsibility of the single monetary policy determined by the ECB. Second, fiscal policy is the responsibility of the individual governments and is constrained by the provisions of the SGP (Stability and Growth Pact). Third, wage developments in relation to productivity are also the responsibility of individual governments and of bargaining between the ,social partners "“ (Allsopp/Artis 2003, S. 4f.).

Geldpolitik soll für Preisstabilität sorgen, nationale Konjunkturdifferenzen sollen durch national zu verantwortende Fiskalpolitik ausgeglichen werden, wobei deren Spielraum durch die Vorgaben des Stabilitäts- und Wachstumspaktes beschränkt wird. Die Verantwortung für stabilitätsgerechte Lohnabschlüsse liegt bei den nationalen Regierungen respektive Sozialpart-

\section{Abb. 4: Arten von Divergenzen}

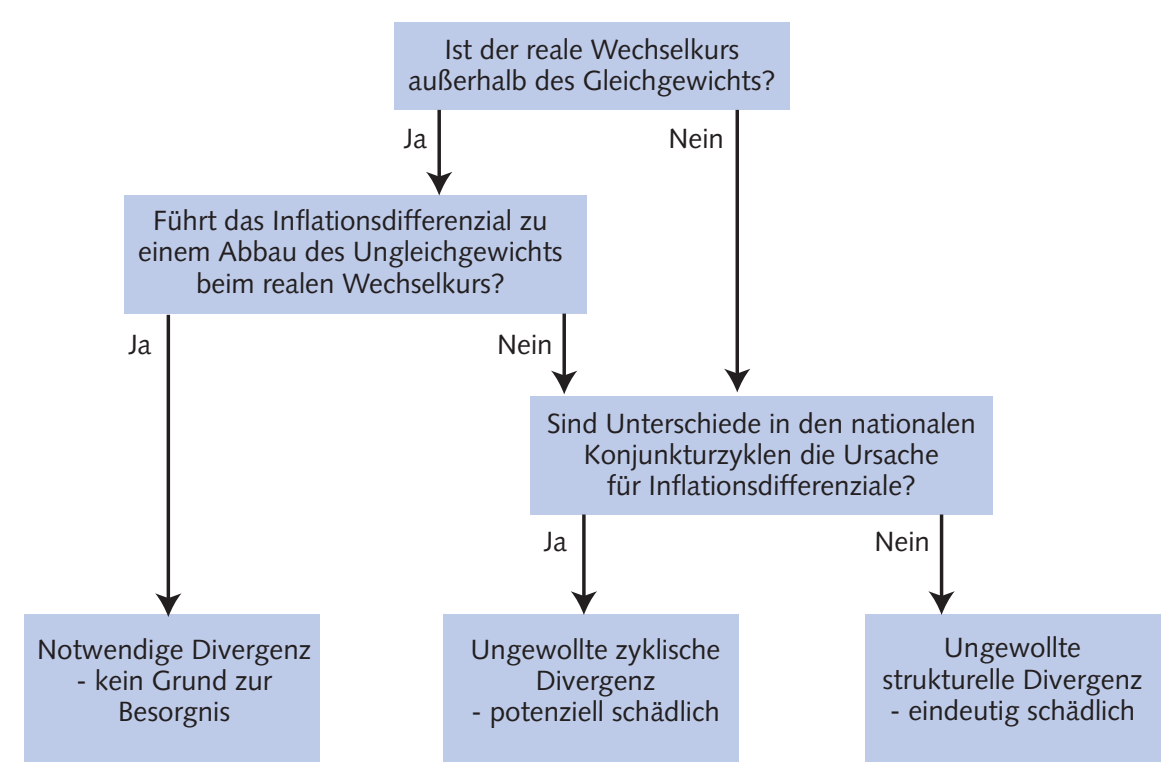

Quelle: Darstellung des Autors.

WSI MITTELIUNGEN

nern. Dahinter steht die Vorstellung, dass die marktendogenen Anpassungsmechanismen innerhalb der Währungsunion hinreichend stark sind, alle beteiligten Parteien stabilitätsgerecht agieren und über die geschilderte Aufgabenteilung der traditionellen konjunkturpolitischen Mechanismen hinaus kein zusätzlicher wirtschaftspolitischer Handlungsbedarf besteht.

Allerdings ist zu beachten, dass die Wirkungen von Anpassungen realer Wechselkurse in einer Währungsunion asymmetrisch sein können, da bei Existenz eines negativen Zusammenhangs zwischen Inflation und Arbeitslosigkeit (Phillips-Kurve) eine reale Abwertung mit deutlich höheren Kosten verbunden ist als eine reale Aufwertung. Insbesondere nach unten starre Nominallöhne, die unter dem Aspekt einer Deflationsverhinderung durchaus sinnvoll erscheinen, machen die Anpassung aus einer Überbewertungssituation mit unwiderruflich fixen Wechselkursen sehr schwierig. Mittelfristig kommt hinzu, dass Hysterese auf Arbeitsmärkten, die sich bei lange anhaltender Arbeitslosigkeit aus Disqualifikation ergeben kann, dazu führen wird, dass auf den ersten Blick „rein konjunkturell“ begründete Entwicklungen hartnäckige Rückwirkungen auf die Entwicklung von Potenzialgrößen haben können.
Aus dieser Logik ergibt sich auch die von Olivier Blanchard schon 2005 geäußerte Warnung, der Euroraum erlebe in den kommenden Jahren „rotierende Krisen“ („rotating slumps“). Bei asymmetrisch auftretenden Schocks träfe es reihum einzelne Länder, die sich in einer für eine gewisse Zeit selbstverstärkenden Krise wiederfänden, welche in der Währungsunion erst sehr langsam überwunden würde (Blanchard 2005, 2006).

An dieser Stelle könnte eingewendet werden, dass selbst bei einem nur langsam wirkendem Ausgleich über den realen Wechselkurs auch von den Realzinsen langfristig Effekte über die Anpassung des Kapitalstocks ausgehen dürften, die in der langfristigen Betrachtung stabilisierend wirken könnten. In Ländern mit unterdurchschnittlichen Realzinsen käme es zu einem deutlichen Kapitalstockaufbau, der wiederum mit einer geringeren Grenzproduktivität zusätzlicher Investitionen einhergeht. Die daraus resultierende geringere Ertragsrate würde zu einer Abschwächung der Investitionsdynamik und letztlich $\mathrm{zu}$ einem Ende des angestoßenen Booms führen. Spiegelbildlich dürfte dieser Effekt in Ländern mit überdurchschnittlichen Realzinsen zu einem Investitionsstau und einem relativen Zurückbleiben in der Kapitalausstattung führen - so lange bis der Grenzertrag der zusätzlichen Investi- 


\section{Abb. 5: Kumulierter Immobilienpreisanstieg (2003-2007) - in \% -}

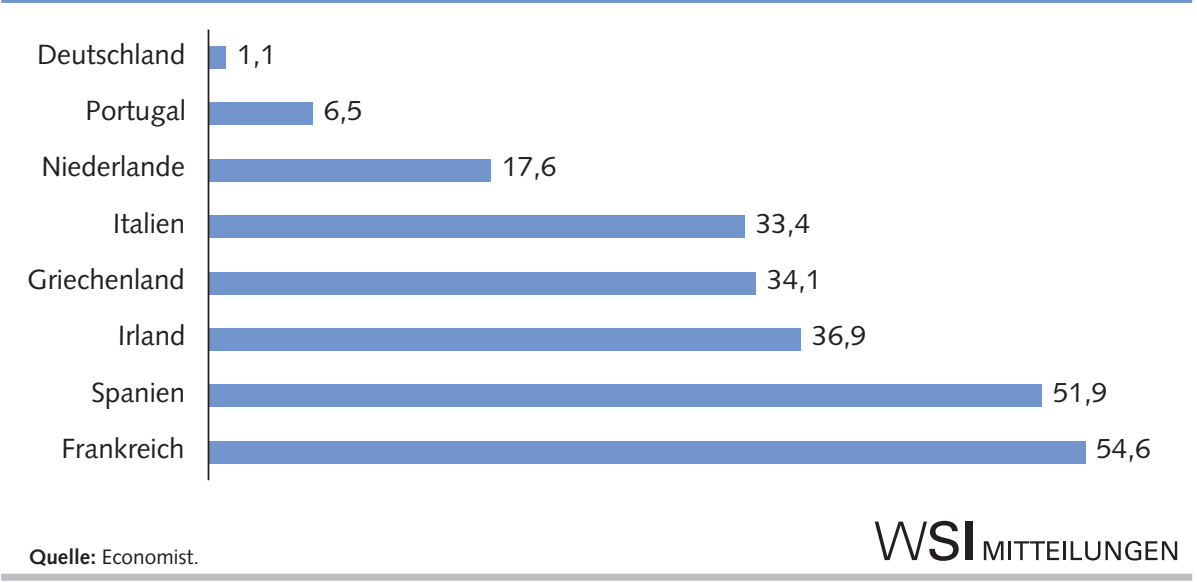

tionen dort so hoch wäre, dass sich selbst hier wieder eine Kapitalstockerhöhung ergäbe. Auf den ersten Blick klingt diese Argumentation durchaus interessant, allerdings funktionieren - wie die derzeitige Krise zeigt und unten noch diskutiert wird - die Anpassungen auf Finanz- und Kapitalmärkten nicht dergestalt, dass in wirtschaftspolitisch relevanten Zeiträumen eine solche Anpassung erwartet werden kann. Überdies wären die ökonomischen wie politischen Folgekosten einer solchen Anpassung enorm.

Abbildung 4 zeigt ein Schema zur Klassifikation von Divergenzen in nützliche, harmlose und schädliche Divergenzen.

Nützlich sind Divergenzen in dem Maße, wie sie zum Abbau eines aufgetretenen Ungleichgewichtes im realen Wechselkurs beitragen. Diese Divergenzen sind notwendiger Bestandteil des Anpassungsprozesses.

Harmlos sind Divergenzen, wenn diese im Zuge von national divergierenden Konjunkturzyklen - als Begleiterscheinung der unterschiedlichen Position im Zyklus auftreten und mittel- bis langfristig nicht $\mathrm{zu}$ einem Auseinanderlaufen der realen Wechselkurse vom Gleichgewicht führen.

Schädlich sind vor allem Divergenzen bei den Lohnstückkosten, die dazu führen, dass sich der reale Wechselkurs über längere Zeit von seinem langfristigen Gleichgewicht entfernt und die gleichzeitig eine Größenordnung erreichen, die deutlich jenseits dessen liegt, was üblicherweise im Laufe eines Konjunkturzyklus erwartet werden könnte. ${ }^{2}$

Im Rahmen eigener empirischer Untersuchungen (Dullien/Fritsche 2008, 2009; Fritsche/Kuzin 2007) wurde untersucht, inwieweit die in den Jahren 2000-2005 zu beobachtende Dispersion in den nationa- len Lohnstückkostenzuwächsen bedenkliche Ausmaße angenommen hat. Als Referenz wurden Vergleichsdaten zu entsprechenden regionalen Entwicklungen in den USA und Deutschland herangezogen. Die Ergebnisse lassen sich in zwei Aussagen zusammenfassen:

(1) Es gibt anhaltende und deutliche Veränderungen in der relativen Lohnstückkostenposition - sowohl in positiver wie negativer Richtung -, die deutlich über das Ausmaß dessen hinausgehen, was in „etablierten“Währungsräumen beobachtbar ist.

(2) Der Anpassungsprozess in Europa ist deutlich langsamer als in den USA oder Deutschland.

Die Ausschläge sind also stärker und die Verwerfungen halten länger an als in etablierten Währungsräumen. Dies deutet darauf hin, dass wir es hier mit schädlichen Lohnstückkostendivergenzen zu tun haben könnten, die ihre tiefere Ursache in einer Störung bei der Ausgestaltung stabilitätsgerechter Lohnpolitik in den Nationalstaaten der Währungsunion haben (Flassbeck/ Spiecker 2009).

Verschärfend wirkt, dass (für längere Zeit) divergierende Inflationsraten mit deutlichen allokativen Verzerrungen bis hin zu Blasen auf Aktien- und Immobilienmärkten einhergehen können und kein institutionalisiertes europäisches Instrumentarium des Krisenmanagements besteht.

Abbildung 5 zeigt für eine Reihe europäischer Länder den kumulativen Preisanstieg im Immobilienbereich für die Zeit von 2003-2007. Auffällig ist, dass sich von den bisher betrachteten Ländern des Eu- roraumes diejenigen mit lang anhaltenden positiven Inflations- und Lohnstückkostenabweichungen (Griechenland, Spanien, Italien, Irland) im „Vorderfeld“ der Immobilienpreisanstiege finden. Spiegelbildlich findet sich Deutschland in der "Schlusslichtposition“, die Immobilienpreise stagnierten hier schlichtweg. Aber auch die Niederlande und Österreich nehmen ,hintere Plätze“ ein.

Einiges deutet darauf hin, dass sich die schnell wachsenden Länder wie Spanien, Irland, Griechenland schon seit einigen Jahren in einer konjunkturellen Überhitzungssituation befunden haben, was sich angesichts der geringen Lohnstückkostenzuwächse („Gürtel enger schnallen“) in Ländern wie Deutschland, Österreich oder in den Niederlanden für den gewichteten europäischen Durchschnitt beim Inflationsziel als folgenlos erwies, aber zu einer Akkumulation von Ungleichgewichten und fragilen Konstellationen im Bereich der Immobilien- und Finanzmärkte in einzelnen Mitgliedsländern beigetragen hat.

Angesichts der derzeitigen krisenhaften Zuspitzung auf den Finanzmärkten hat die Diskussion über mögliche Ungleichgewichte in einer Währungsunion eine neue Dynamik erhalten. Nun sind insbesondere diejenigen Länder mit den ausgeprägten Leistungsbilanzdefiziten (und zum Teil geplatzten Immobilienblasen) - hier vor allem Griechenland, Portugal, Spanien, Irland - mit deutlichen Zinsaufschlägen und einer Verschlechterung des Kreditratings

\footnotetext{
2 Nun wäre es auch denkbar, dass der kräftige Anstieg der relativen Lohnstückkosten in Ländern wie Portugal oder Spanien ein Gleichgewichtsphänomen wäre. Da in den Volkswirtschaften mit aufholender Entwicklung mehr in neue Ausrüstungen investiert wird, steige die Arbeitsproduktivität vor allem im verarbeitenden Gewerbe kräftig, so wird argumentiert. Die Arbeitskräfte in diesem Sektor profitierten davon durch steigende Löhne. Da sich die Arbeitskräfte in den anderen Sektoren mit nicht-handelbaren Gütern (Dienstleistungen, Bau, etc.) an dieser Lohnentwicklung orientierten, ziehe das Lohnwachstum in der ganzen aufholenden Wirtschaft stärker als die Durchschnittsproduktivität an und treibe die Inflation in die Höhe. Tatsächlich ist aber seit Beginn der Währungsunion die Produktivität in Spanien, Italien und Portugal auch für handelbare Güter deutlich langsamer gestiegen als im Rest der Eurozone oder im reicheren Deutschland (Langedijk/Roeger 2007). Ein besonders starkes Produktivitätswachstum der Industrie dieser Länder ist damit als Ursache für den höheren Lohnanstieg nicht plausibel (Lommatzsch/ Tober 2006). Auch der Verlust von Exportmarktanteilen dieser Länder deutet auf ein Problem der Wettbewerbsfähigkeit hin.
} 
konfrontiert, was ganz aktuell folgende Fragen aufwirft: Wie hoch ist das Ausmaß von Ungleichgewichten, das unter den bestehenden institutionellen Rahmenbedingungen der Wirtschafts- und Währungsunion aushaltbar ist? Wie kann eine aktuelle Krisenbewältigungsstrategie aussehen? Und wie kann eine funktionierende wirtschaftspolitische Strategie aussehen, die derartige Entwicklungen entweder dämpft oder von vornherein ausschließt?

Die bisher vorliegenden Untersuchungen - die weit davon entfernt sind eine abschließende Bewertung der Anpassungsprozesse zu ermöglichen - geben zumindest deutlichen Anlass zur Sorge bezüglich der Höhe der Ungleichgewichte. Sowohl das Anwachsen der Leistungsbilanzdefizite als auch die Verschiebung der realen Wechselkurse über die vergangenen zehn Jahre waren in ihren Ausmaßen sehr deutlich und wahrscheinlich historisch einmalig. Offenbar sind marktendogene Ausgleichsmechanismen zu schwach, um in kürzeren Zeiträumen eine Anpassung herbeizuführen. Diese Ergebnisse dämpfen den Optimismus, der dem genannten „consensus view“ hinsichtlich der Aufgabenzuordnung der wirtschaftspolitischen Instrumente zugrunde liegt, deutlich. Zahlreiche Resultate sowohl der EU-Kommission (DG ECFIN 2008) als auch der EZB (Angeloni et al. 2006) deuten darauf hin, dass die Trägheit der Anpassungsprozesse und die damit verbundenen Friktionen im Euroraum eine andere Dimension haben als z. B. in den USA und nach einem wirtschaftspolitischen Eingriff verlangen. Allerdings unterscheiden sich die Lösungsvorschläge deutlich hinsichtlich der Konzepte, die dabei verfolgt werden.

\section{Lösungsvorschläge}

Grundsätzlich besteht in einer Währungsunion natürlich die Möglichkeit, dass sich Ungleichgewichte marktvermittelt oder bei Vorliegen von Marktversagen durch den Einsatz geeigneter wirtschaftspolitischer Instrumente abbauen lassen. Der von Allsopp/Artis (2003) als „consensus view“ beschriebene Ansatz setzt dabei im Kern auf den Markt als Ausgleichmechanismus, wobei insbesondere in den ersten Jahren der Währungsunion das Vertrauen in die "Selbstheilungskräfte" marktwirtschaftlicher Prozesse bei gleichzeitiger starker
Selbstbindung der nationalen Fiskalpolitiken durch die engen Vorgaben des Stabilitäts- und Wachstumspaktes dogmatischen Charakter trug.

Die vergangenen Jahre haben deutlich gezeigt, dass sich dieser Optimismus bezüglich der Selbstheilungskräfte des Marktes nicht halten ließ. Der Marktmechanismus führt auch nicht automatisch dazu, dass der verteilungsneutrale Spielraum der Lohnpolitik ausgeschöpft wird. Die Divergenzen waren und sind deutlich und haben Spuren hinterlassen. Insbesondere hat sich gezeigt, dass die Finanzkrise des Jahres 2008 die nationalen Volkswirtschaften des Euroraumes in ganz unterschiedlicher Weise betroffen hat, was in engem Zusammenhang zu den Inflations- und Lohnstückkostendivergenzen steht.

Auf der einen Seite traf die Finanzkrise eine ganze Reihe von Ländern wie Spanien, Griechenland und Irland in einer wirtschaftlichen Lage mit hohen Leistungsbilanzdefiziten und einer Immobilienmarktsituation, die durch eine sehr hohe Bewertung gekennzeichnet war. Diese Länder hatten lange Zeit mit überdurchschnittlich hohen Wachstumsraten bei zugleich überdurchschnittlichen Inflationsraten expandiert, gleichzeitig über die vergangene Dekade aber sehr stark an Wettbewerbsfähigkeit verloren. Das Herdenverhalten von Investoren führte dazu, dass in die sich aufblähende Immobilienblase hinein investiert wurde, wobei auf unrealistischen Bewertungen basierende scheinbare Vermögenseffekte den privaten Konsum zusätzlich anheizten.

Auf der anderen Seite standen Länder wie Deutschland oder Österreich, die in den vergangenen Jahren niedrige Inflationsraten und eine deutliche Verbesserung der relativen Lohnstückkostenposition bei exportgetragenem Wachstum erreicht hatten. Insbesondere in Deutschland ging die Entwicklung der letzten Dekade einher mit einer deutlich durch Abwertungserfolge exportorientierten Entwicklung bei einer gleichzeitig schwachen Binnenkonjunktur (Carlin/Soskice 2008). Die Ironie der Geschichte dabei ist, dass diese Konstellation zwar dafür sorgte, dass für den EuroraumDurchschnitt die Inflationsentwicklung mäßig blieb, jedoch die nun überfällige Korrektur der Immobilienblase mit voller Wucht auf die stark von exportgetragenem Wachstum abhängigen Länder zurückfällt.

In der jetzigen Situation befinden sich beide Ländergruppen in einem Dilemma.
Die einen bräuchten eine deutliche Verbesserung der Wettbewerbsfähigkeit, um bei wegbrechender Binnennachfrage die Wirtschaft zu stabilisieren, den anderen nützt die beste Wettbewerbsfähigkeit nichts, wenn die Auslandsnachfrage wegbricht. Eine Verbesserung der Situation könnte durch eine Bereinigung der Fehlentwicklung bei den realen Wechselkursen herbeigeführt werden. Theoretisch ist der Fall recht klar, wie aber praktisch in einer Währungsunion eine deutliche Korrektur von Ungleichgewichten des realen Wechselkurses ohne Anpassung nominaler Wechselkurse herbeigeführt werden kann, ist eine historisch bislang wenig getestete Frage (Eichengreen 2007). Ländern wie Spanien wird letztlich nicht viel anderes übrig bleiben, als durch Lohnmoderation bei einer gleichzeitig deutlichen Steigerung der Produktivitätsentwicklung ein Zurückbleiben in der Lohnstückkostenentwicklung herbeizuführen (Blanchard 2006). Dies ist jedoch ein sehr steiniger und mühevoller Weg. Zudem besteht die Gefahr, dass es zu einem Deflationswettbewerb kommt, da eine negative Inflationsrate sowohl die Erholung des Finanzsystems stark belastet (Fisher 1933) als auch zu einer „wait-and-see“-Haltung bei Konsumund Investitionsausgaben führen kann. Eine Korrektur der relativen realen Wechselkurse im Euroraum sollte deshalb auch über eine deutliche reale Aufwertung derjenigen Länder erfolgen, die relativ zum Euroraum-Durchschnitt geringere Lohnsteigerungen verzeichneten und ihre Wettbewerbsposition auf Kosten der anderen Länder verbesserten („Beggar-thy-neighbour"-Politik). Für Länder wie Deutschland wäre eine relative Inflationssteigerung (für einige Zeit überdurchschnittliche Inflationsraten relativ zum Euroraum) als Beitrag zur Stabilisierung der Situation im Euroraum deshalb nur anzuraten - wenngleich schwierig umsetzbar.

Neben dem „Tagesgeschäft", das in der jetzigen Situation vieles überlagert, ist auch zu diskutieren, durch welche institutionellen Reformen und wirtschaftspolitischen Weichenstellungen ein reibungsloses Funktionieren der Währungsunion in der Zukunft erreicht werden könnte. Die in der Vergangenheit von der EU-Kommission wie auch der Europäischen Zentralbank (EZB) offiziell gemachten Ratschläge laufen vor allem auf eine Stärkung der Faktorund Produktmarktflexibilität hinaus (Trichet 2006; DG ECFIN 2008). Dabei ist ge- 
gen eine Stärkung wettbewerblicher Kräfte aus allokationspolitischen Gesichtspunkten in vielen Fällen nichts einzuwenden. So kann von einer schnelleren Anpassung von Produktivitätsunterschieden auf europäischer Ebene ein schnellerer Abbau der Ungleichgewichte erwartet werden (Blanchard 2005, 2006). Ob jedoch allein daraus eine Verbesserung der Anpassungskapazität auf europäischer Ebene im Sinne einer Verminderung der Inflationsdivergenzen möglich ist, ist fraglich. Dies vor allem deshalb, weil auf Produkt- wie Faktormärkten jede stärkere Liberalisierung gewollte oder ungewollte Nebeneffekte hat, die es zu bedenken gilt. Neben einer deutlichen Erhöhung der Arbeitskräftemobilität, die im europäischen Rahmen sowohl politisch schwierig als auch aus Gründen existierender Sprachbarrieren kaum realistisch erscheint, würde eine geforderte deutliche Nominallohnflexibilität auch die Gefahr einer sich verstärkenden Deflationsspirale in sich bergen, die mit anhaltenden stagnativen Tendenzen verbundenen wäre.

Deshalb muss überlegt werden, welche wirtschaftspolitischen Anpassungen erfolgen könnten, um die Funktionsfähigkeit des Euroraums zu stärken. Hier hilft ein Verweis auf die Erfahrungen in etablierten Währungsräumen wie den USA oder Deutschland. Während die Geldpolitik auf europäischer Ebene kaum geeignet erscheint, nationale Differenzen in den Inflationsraten auszugleichen, da der kurzfristige Zinssatz als Hauptinstrument der Geldpolitik für alle Staaten des Euroraums gleich ist, könnte die Fiskalpolitik eine wichtige Rolle spielen. Zwei grundsätzliche Strategien bieten sich an: Zum einen könnte darauf hingearbeitet werden, ein effektiveres Gegensteuern der Fiskalpolitik bei Fehlentwicklungen auf nationaler Ebene durch ein konsequentes Ausschöpfen der fiskalpolitischen Spielräume zu ermöglichen, vor allem was die Bildung von Überschüssen in Ländern mit einem deutlichen
Überschreiten des Inflationsziels anbetrifft. Die Erfahrung der vergangenen Jahre zeigte, dass ein solcher (frommer?) Wunsch zwar nachvollziehbar ist, jenseits wirtschaftspolitischer Absichtsbekundungen aber nur schwer umsetzbar erscheint. Deshalb könnte zum anderen ein (konjunkturpolitischer!) Finanzausgleich zwischen Ländern in unterschiedlichen Konjunktursituationen effektiv zu einer Dämpfung der Ausschläge in der Wirtschaftsaktivität und damit auch des Inflationsdrucks führen. Eine solche Stärkung der automatischen Stabilisatoren auf der Ebene zwischen den Mitgliedstaaten könnte unterschiedlich aussehen. Für eine zentralisierte Fiskalpolitik in der EWU existieren zahlreiche Vorschläge, u.a. die Nutzung des EU-Budgets für einen Ausgleich zyklischer Schwankungen (vgl. Beitrag von Dullien/Schwarzer in diesem Heft), eine europaweite europäische Arbeitslosenversicherung, die als eine Art konjunktureller Länderfinanzausgleichsmechanismus auf europäischer Ebene wirken könnte (Dullien 2008), oder die etwas traditionellere Idee eines europäischen Transferfonds (Pisani-Ferry et al. 1993).

Allerdings setzen auch diese Vorschläge ein hohes Maß an politischem Gestaltungswillen auf europäischer Ebene voraus, der in dieser Form derzeit nicht erkennbar ist. Deshalb gibt es zwei andere Vorschläge, die ohne diesen hohen politischen Aufwand kurzfristig umsetzbar sind:

Zum einen geht es um die Stärkung der Koordinierung der europäischen Fiskalpolitik über eine Stärkung der „Euro-Group“ (Solow et al. 2009), da dies schnell erreichbar scheint und angesichts der derzeitigen krisenhaften Entwicklung die dringend notwendige Handlungsfähigkeit auf europäischer Ebene stärkt. Kurzfristig gilt es, eine „Beggar-thy-neighbour“-Politik bei den nationalen Konjunkturprogrammen zu vermeiden. Mittelfristig käme einer derartig koordinierten Finanzpolitik dann vor allem die Aufgabe zu, nicht nur konjunkturelle Schwächephasen durch expansive Anstrengungen aller europäischen Länder auszugleichen, sondern vielmehr dafür zu sorgen, dass Inflationsratendifferenzen durch eine restriktive Finanzpolitik (Bildung von Überschüssen, Entschuldung) in den Leistungsbilanzdefizitländern bzw. eine expansive Fiskalpolitik in den Überschussländern abgebaut werden.

Zum anderen sollte dringend ein Prozess der europäischen Lohnkoordinierung in Gang gebracht werden; darauf haben vor allem Koll/Hallwirth (2009, sowie Beitrag in diesem Heft), Hallwirth (2005) und Collignon (2009) hingewiesen. Wie Collignon (2009) zeigt, scheint eine an (europäischem) Inflationsziel und trendmäßigem nationalen Produktivitätszuwachs orientierte Lohnpolitik in vielen europäischen Ländern nicht zu funktionieren. Eine stärkere Koordinierung könnte dafür sorgen, das Problembewusstsein für das Entstehen und die langfristigen Nebeneffekte anhaltender und deutlicher Inflationsdifferenzen zu stärken. Letztlich sind die langfristigen Anpassungskosten auch aus Arbeitnehmersicht deutlich höher zu veranschlagen als die kurzfristigen Gewinne aus einer „Beggar-thy-neighbour“-Strategie. In dem Maße, wie eine koordinierte Lohnpolitik gelingt, könnten lang anhaltende Divergenzprozesse bei den Lohnstückkosten wie in Spanien oder Deutschland gestoppt und die sich aufschaukelnden und letztlich schmerzhaften (mit hohen Arbeitsplatzverlusten verbundenen) Anpassungsprozesse gebrochen werden. In Ländern wie Spanien und Portugal sollte zudem jegliche noch vorhandene Form der expliziten wie impliziten Indexierung von Lohnverträgen abgeschafft werden. Zusätzlich könnte darüber diskutiert werden, staatliche Anreize zu setzen, um stabilitätsorientierte Lohnkontrakte einzuhalten, z. B. durch zeitlich befristete Steuererleichterungen. 


\section{LITERATUR}

Alesina, A./Blanchard, O./Gali, J./Giavazzi, F /Uhlig, H. (2001): Defining a Macroeconomic Framework for the Euro Area, Monitoring the European Central Bank 3, CEPR

Allsopp, C./Artis, M. (2003): The Assessment: EMU, Four Years on, Oxford Review of Economic Policy 1, S. 1-29

Angeloni, I./Aucremanne, L./Ehrmann, M./Gali, J./Levin, A./Smets, F. (2006): New evidence on inflation persistence and price stickiness in the Euro area: Implications for macro modelling, in: Journal of the European Economic Association 4, S. 562-574

Belke, A./Gros, D. (2007): Instability of the Eurozone? On Monetary Policy, House Prices and Labor Market Reforms, Institute for the Study of Labor (IZA), IZA Discussion Papers 2547

Blanchard, O. (2005): A Macroeconomic Survey of Europe, Mimeo, http://econ-www.mit.edu/faculty/blanchar/papers

Blanchard, O. (2006): Adjustment within the Euro: The difficult case of Portugal, Massachusetts Institute of Technology, Department of Economics, Working Paper 06-04

Campbell, J./Mankiw, N. G.(1989): Consumption, Income, and Interest Rates: Reinterpreting the Time-Series Evidence, in: Blanchard, O./Fischer, S. (Hrsg.): NBER Macroeconomics Annual, S. 185-216

Carlin, W./Soskice, D. (2008): German Economic Performance: Disentangling the Role of Supply-side Reforms, Macroeconomic Policy and Coordinated Economy Institutions, Socio-Economic Review, doi: 10.1093/ser/mwn021, http://tinyurl.com/4nz43y

Collignon, S. (2009): Wage developments in Euroland or: The Failure of the Macroeconomic Dialogue, Mimeo, Sant' Anna School of Advanced Studies, Pisa

Dullien, S. (2008), Eine Arbeitslosenversicherung für die Eurozone: Ein Vorschlag zur Stabilisierung divergierender Wirtschaftsentwicklungen in der Europäischen Währungsunion, SWP-Studie S01, Berlin Dullien, S./Fritsche, U. (2008): Does the Dispersion of Unit Labor Cost in the EMU imply long-run Convergence?, in: International Economics and Economic Policy 3, S. 269-295

Dullien, S./Fritsche, U. (2009): How bad is Divergence in the Euro-Zone? Lessons from the United States of America and Germany, in: Journal of Post Keynesian Economics, im Erscheinen

Dullien, S./Schwarzer, D (2005): The Euro-Zone under High Pressure, Comment, Stiftung Wissenschaft und Politik, May, http://www. swpberlin.org/common/get document.php?id=1298

Dullien, S./Schwarzer, D. (2009): Bringing Macroeconomics into the EU Budget Debate: Why and How?, in: Journal of Common Market Studies 1, S. 153-174

Eichengreen, B. (2007): The Breakup of the Euro Area, NBER Working Paper 13393, Cambridge, MA
Europäische Kommission, Generaldirektion Wirtschaft und Finanzen (DG ECFIN) (2008): EMU@10. Successes and challenges after ten years of Economic and Monetary Union, European Economy 2

Fisher, I. (1933): The Debt-Deflation Theory of the Great Depressions, in: Econometrica 1, S. 337-357

Flassbeck, H./Spiecker, F. (2009): Zwischen Pest und Cholera?, in: Wirtschaftsdienst 3, S. 142-143

Fritsche, U./Kuzin, V. (2007): Unit labor cost growth differentials in the Euro area, Germany, and the US: lessons from PANIC and cluster analysis, DEP Discussion Papers, Macroeconomics and Finance Series 3

Geiger, F./Spahn, H.-P. (2007): One size fits none? Monetary Policy and Inflation Differentials in EMU, in: Hein, E./Priewe, J./Truger, A. (Hrsg.):

European Integration in Crisis, Marburg

Gros, D. (2006): Will EMU survive 2010?, Policy brief, Centre for European Policy Studies, http://www.ceps.be/Article.php?article_id=503 Hallwirth, V. (2005): Beschäftigungsorientierte Lohnpolitik, in: Wirtschaftsdienst 5, S. 295-303

Issing, O. (2002): On Macroeconomic Policy Co-ordination in EMU, in: Journal of Common Market Studies 2, S. 345-358

Koll, W./Hallwirth, V. (2009): Zehn Jahr Makroökonomischer Dialog Eine Zwischenbilanz, in: Wirtschaftsdienst 1, S. 26-33

Lane, P. (2006): The real effects of EMU, CEPR, Discussion Paper Series 5536

Langedijk, S./Roeger, W. (2007): Adjustment in EMU. A model-based analysis of country experiences, European Economy, Economic Papers 274

Lommatzsch, K./Tober, S. (2006): Euro-Area Inflation - Does the BalassaSamuelson Effect matter?, in: International Economics and Economic Policy 2, S. 105-136

Münchau, W. (2006): Spain has reason to quit the euro, Financial Times, February 19

Pisani-Ferry, J./Italianer, A./Lescure R. (1993): Stabilization properties of budgetary systems: A simulation analysis, in: European Economy, Reports and Studies 5, S. 511-538

Prior-Wandesforde, R./Hacche, G. (2005): European Meltdown? Europe fiddles as Rome burns, HSBC Global Research European Economics, London

Solow, R. et al (2009): Towards a Common Fiscal Policy: Relaunching the Eurozone Economies, Appeal launched by the Cournot Centre and its president Nobel Laureate Robert Solow (MIT), http://www.centrecournot. org/

Trichet, J. C. (2006): Economic integration in the euro area. Speech by Mr Jean-Claude Trichet, President of the European Central Bank, at the 15th European Regional 673 Conference of the Board of Governors, Tel Aviv University, Paris, 31. March, BIS Review 27, S. 1-7 